\title{
Statistical Properties of Single-Mode Displaced Squeezed Vacuum State
}

\author{
Alemayehu Getahun Ruth Birhanu \\ Department of physics, Mekdela Amba University, P.O.Box 32, Tullu Awulia, Ethiopia
}

\begin{abstract}
In this paper the displaced squeezed vacuum state is produced by application of displaced operator on squeezed vacuum state. With help of density operator we find $Q$ function, with the $Q$ function mean, variance and quadrature variance would be calculated. From this we can determine the system has superpoissonian statics, the squeezed parameter is direct proportion with both mean and variance of photon number, but inversely proportion with quadrature variance. The squeezing occurs in plus quadrature with the maximum squeezing of $99.7 \%$ for $r=3$.
\end{abstract}

Keywords: Quantum nature, displaced state, squeezed vacuum state

DOI: $10.7176 /$ APTA/81-02

Publication date: December $31^{\text {st }} 2019$

\section{Introduction}

Squeezed states of light have been observed in a variety of quantum optical systems, which are used to enhance the measurement sensitivity in, opt mechanics [1], and even in biology [2]. In squeezed states of light, the noise of the electric field at certain phases falls below that of the vacuum state. This means that, when we turn on the squeezed light, we see less noise than no light at all. This apparently paradoxical feature is a direct consequence of quantum nature of light and cannot be explained within the classical framework [3].

They are described interims of single-mode, two-mode and as the mixtures with the other quantum states of light. The single-mode squeezed light is produced by a degenerate parametric amplifier, consisting of a nonlinear crystal pumped by coherent light. And the two-mode squeezed light is generated by a non-degenerate subharmonic system consisting of nonlinear crystal pumped by coherent light. Two-mode squeezed vacuum state is defined by applying the two-mode squeezed operator to the two-mode vacuum state. We can use the squeezed states of light with mixing them with other quantum states of light. The displaced number state is obtained by application of squeezing operator on displaced and number states [4-9].

In this paper we seek to determine the quantum nature of displaced squeezed vacuum state. We obtained it by application of displaced states on the squeezed vacuum, and by calculating its density operator we determine its quantum nature as described below.

\section{Displaced Squeezed vacuum state}

\subsection{Single-mode squeezed vacuum state}

Single-mode squeezed vacuum state is the prototype of a degenerate parametric amplifier consists of nonlinear crystals pumped by coherent light [10]. The Hamiltonian for degenerate parametric amplifier is given by,

$$
H=\frac{i \in}{2}\left(\hat{a}^{2}-\hat{a}^{+2}\right) \text {. }
$$

The state vector of light for single-mode light initially in coherent state $\mid \gamma>$ can be expressed as

$|\varphi(r)>=\hat{S}(r)| \gamma>$,

Where the squeezed operator takes the form

$\hat{S}(r)=e^{\frac{r}{2}\left(\hat{a}^{2}-\hat{a}^{+2}\right)}$,

To this end, we can express the operator $a(r)$ by;

$a(r)=\hat{S}^{+}(r) a \hat{S}(r)$,

Differentiating the above equation wrt $\mathrm{r}$ and using $I=\hat{S}^{+}(r) \hat{S}(r)$, Eq. (4) takes the form

$a(r)=\hat{a} \cosh r-\hat{a}^{+} \sinh r$

On account of Eqs. (5), (4) and Eq. (3) we can re write the state vector for single-mode squeezed vacuum light as; $|\varphi(r)>=\hat{S}(r)| 0>$.

To this end, we can write the displaced squeezed vacuum state in the form of [11]

$$
|\alpha, r>=\widehat{D}(\alpha) \hat{S}(r)| 0>\text {. }
$$

Where

$\widehat{D}(\alpha)=\exp \left[\alpha \hat{a}^{+}-\alpha^{*} \hat{a}\right]$,

From this we can easily determine the density operator for displaced squeezed vacuum state as

$\hat{\rho}_{d s v}=\widehat{D}(\alpha) \hat{S}(r) \hat{\rho}_{0} \widehat{D}^{+}(\alpha) \hat{S}^{+}(r)$.

Where $\hat{\rho}_{0}=|0><0|$ is density operator for vacuum state 


\section{The $Q$ Function}

The $\mathrm{Q}$ function is defined in [12] as

$Q\left(\alpha^{*}, \alpha, r\right)=\frac{1}{\pi} \int d^{2} \gamma \varphi_{a}\left(\gamma^{*}, \gamma, r\right) \mathrm{e}^{\left(\gamma^{*} \alpha-\gamma \alpha^{*}\right)}$.

Where

$\varphi_{a}\left(\gamma^{*}, \gamma, r\right)=\operatorname{Tr}\left(\hat{\rho}_{d s v} e^{\alpha \hat{a}^{+}} e^{-\alpha^{*} \hat{a}} e^{\gamma a(r)} e^{-\gamma \hat{a}^{+}}\right)$

Applying Backer Huasdrof identity

$$
e^{A} e^{B}=e^{A+B+\frac{1}{2}[A, B]} \text {. }
$$

Eq. (11) takes the form

$$
\varphi_{a}\left(\gamma^{*}, \gamma, r\right)=\frac{1}{\pi} e^{-y \gamma^{*} \gamma+\frac{x}{2}\left(\gamma^{2}+\gamma^{* 2}\right)} .
$$

Where

$\mathrm{y}=\cosh ^{2} \mathrm{r}$.

$x=-\cosh r \sinh r$

Substituting Eq. (13) into Eq. (10) we see that

$Q\left(\alpha^{*}, \alpha, r\right)=\frac{1}{\pi} \int \frac{d^{2} \gamma}{\pi} e^{\left(-y \gamma^{*} \gamma+\gamma^{*} \alpha-\gamma \alpha^{*}+\frac{x}{2}\left(\gamma^{* 2}+\gamma^{2}\right)\right)}$.

Carrying out integration with help of [6], the Q function for displaced squeezed vacuum state takes the form

$Q\left(\alpha^{*}, \alpha, r\right)=\frac{\left(u^{2}-v^{2}\right)^{\frac{1}{2}}}{\pi} \exp \left[-u \alpha^{*} \alpha+v\left(\alpha^{2}+\alpha^{* 2}\right)\right]$.

Where

$u=\frac{y}{y^{2}-x^{2}}$, and $v=\frac{x}{y^{2}-x^{2}}$.

\section{Photon Statistics}

Here we seek to calculate the mean and variance of photon number

\section{1 mean photon number}

Mean photon number is described in terms of Q function as

$$
\bar{n}=\int d^{2} \alpha Q\left(\alpha^{*}, \alpha, r\right)\left[(1-u)^{2} \alpha^{*} \alpha+v \alpha^{2}\right]
$$

$=(1-u) R_{1}+v R_{2}$

Where

$R_{1}=\int d^{2} \alpha Q\left(\alpha^{*}, \alpha, r\right) \alpha^{*} \alpha$

And

$R_{2}=\int d^{2} \alpha Q\left(\alpha^{*}, \alpha, r\right) \alpha^{2}$

Using Eq. (17) into Eq. (20) and (21) and carrying out integration wrt $\alpha$ we get

$R_{1}=y=\cosh ^{2} r$

$R_{2}=x=-\cosh r \sinh r$.

On account of Eq. (22) and (23) into Eq. (19) and using trigonometric identity we set the mean photon number for displaced squeezed vacuum state as;

$\bar{n}=\sinh ^{2} r$.

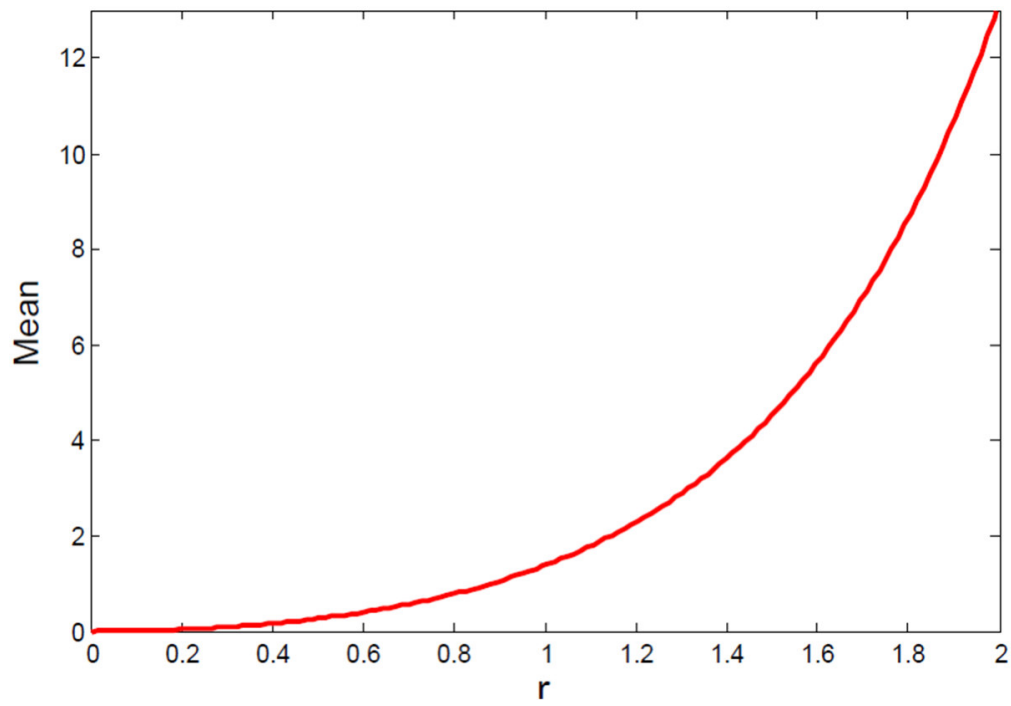


Figuare 1: Plots of mean photon number Eq. (24) versus squeeze parameter( $r$ )

Fig. (1) shows the relation between mean of photon number with squeezed parameter, and we can understand that both mean photon number and squeezed parameter have the directly proportional.

\subsection{The Variance of Photon Number}

The variance of photon number for single-mode state is settled in anti-normal order form as; $(\Delta n)^{2}=<\hat{a}^{+2} \hat{a}^{2}>+2 \bar{n}-\bar{n}^{2}$.

Where

Where

$$
\begin{gathered}
<\hat{a}^{+2} \hat{a}^{2}>=\int d^{2} \alpha Q\left(\alpha^{*}, \alpha, r\right)\left[(1-u) \alpha^{* 2} \alpha^{2}+v \alpha^{2}+2 v(1-u) \alpha^{*} \alpha^{3}+v^{2} \alpha^{2}\right] \\
=v I_{1}+(1-u)^{2} I_{2}+2 v(1-u) I_{3}+v^{2} I_{4}
\end{gathered}
$$

$I_{1}=\int d^{2} \alpha Q\left(\alpha^{*}, \alpha, r\right) \alpha^{2}$

$I_{2}=\int d^{2} \alpha Q\left(\alpha^{*}, \alpha, r\right) \alpha^{* 2} \alpha^{2}$,

$I_{3}=\int d^{2} \alpha Q\left(\alpha^{*}, \alpha, r\right) \alpha^{*} \alpha^{3}$,

And

$\mathrm{I}_{4}=\int \mathrm{d}^{2} \alpha \mathrm{Q}\left(\alpha^{*}, \alpha, \mathrm{r}\right) \alpha^{4}$.

Substituting Eq. (17) into all of Eqs. (27) And carrying out integration wrt. After substituting values of $I_{1}, I_{2}, I_{3}$ and $I_{4}$ Eq. (26) takes the form;

$<\hat{a}^{+2} \hat{a}^{2}>=2 y^{2}+x^{2}$.

Using Eqs. (14) And (15) into Eq. (28) and with the help of Eq. (24) we can put the variance of photon number as; $(\Delta n)^{2}=3 \sinh ^{2} r+2 \sinh ^{4} r-\cosh r \sinh r$.

Fig.(2) Shows the variance of photon number is greater than mean photon number, so the radiation of displaced squeezed vacuum state is super-posonian photon statics.

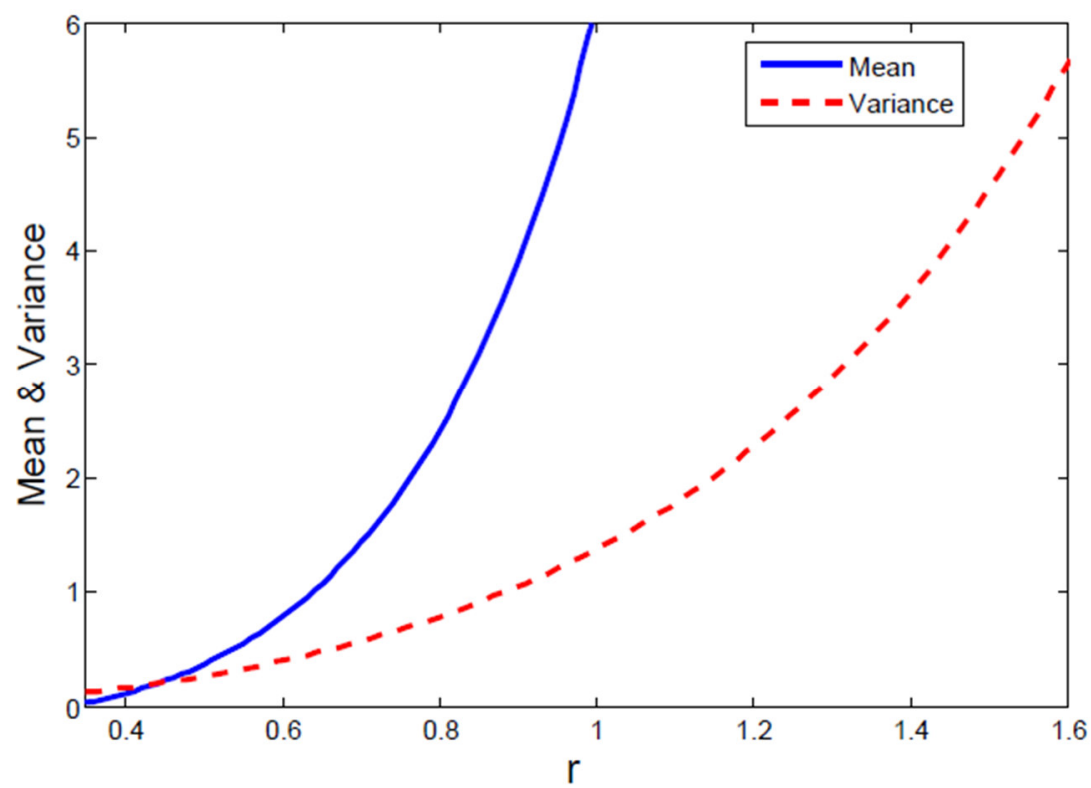

Figure 2: plots of mean (Eq. (24)) and variance of photon number (Eq. (29))

\subsection{Quadrature squeezing}

Quadrature squeezing is used to determine the squeezing properties of the given light. The single-mode light is said to be squeezed light if $\Delta a_{+}<1$ or $\Delta a_{-}<1$, and it must satisfies $\Delta a_{+} \Delta a_{-} \geq 1$. Where plus and minus quadrature variance can be related by

$$
\widehat{a_{ \pm}}=\sqrt{ \pm 1}\left(\hat{a}^{+} \pm \hat{a}\right) \text {. }
$$

Which must satisfies the commutation relation of

$$
\left[a_{+}, a_{-}\right]=2 \mathrm{i} \text {, }
$$

Then one can describe the quadrature variance depend on the above equation as;

$\Delta a_{ \pm}^{2}=<\widehat{a_{ \pm}}, \widehat{a_{ \pm}}>$,

On account of Eq. (30) the above equation takes the form of;

$$
<\Delta \widehat{a_{ \pm}}>^{2} \quad=1 \quad \pm<\hat{a}^{2}> \pm<\hat{a}^{+2}>+2<\hat{a}^{+} \hat{a}>\mp<\hat{a}^{+}>^{2}-2<\hat{a}><\hat{a}^{+}>\text {. }
$$


After evaluating all expectation values in above equation the quadrature variance of displaced squeezed vacuum takes the form;

$<\Delta \widehat{a_{ \pm}}>^{2}=2 \sinh ^{2} r+1 \mp 2 \cosh r \sinh r$.

Expressing the trigonometric function interims of exponential notation Eq. (34) takes the form

$<\Delta \widehat{a_{ \pm}}>^{2}=e^{\mp 2 r}$.

Eq. (35) is the quadrature variance for displaced squeezed vacuum state. From this equation we can easily states that the squeezing occurs in plus quadrature.

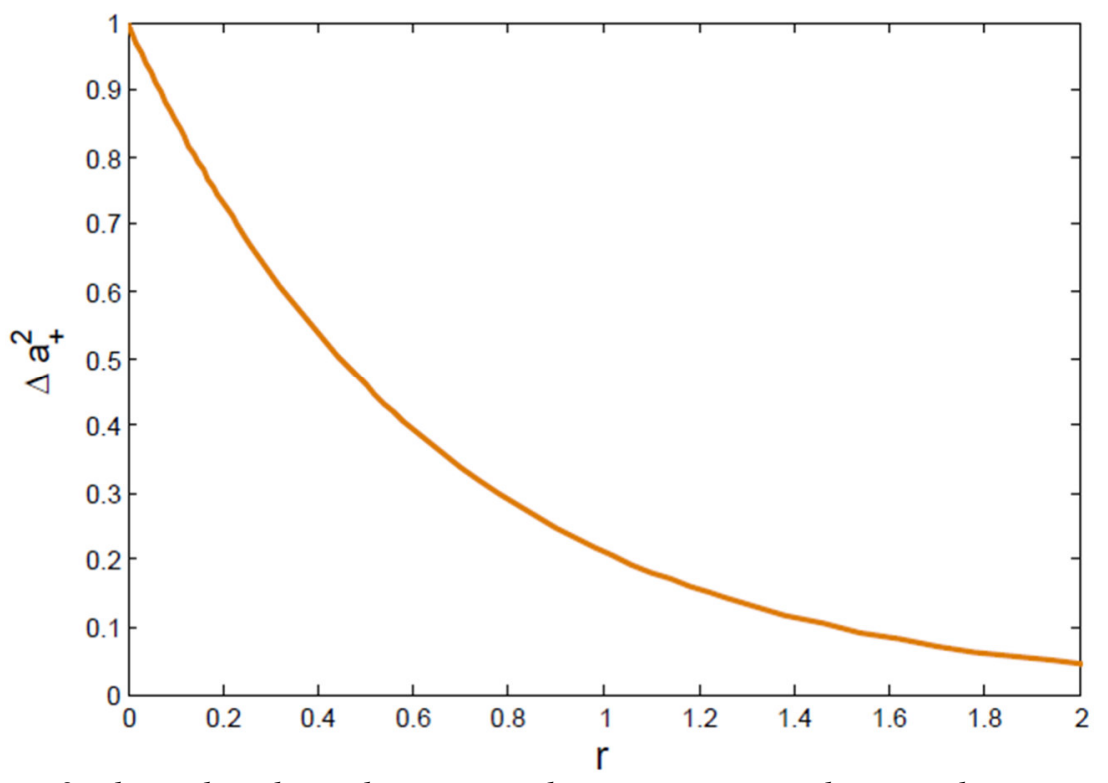

Figure 3: shows the relation between quadrature variance and squeezed parameter.

Fig (3) shows the quadrature variance and squeezed parameters are inversely proportional. The other thing is that the graph of quadrature variance does not extend above 1 . This indicates the calculation full fill the squeezing properties.

To this end, we can drive quadrature squeezing relative to vacuum state as;

$s=\frac{\left(\Delta a_{ \pm}^{2}\right)_{v}-\Delta a_{+}^{2}}{\left(\Delta a_{ \pm}^{2}\right)_{v}}$

We can calculate $\left(\Delta a_{ \pm}{ }^{2}\right)_{v}$ from Eq. (35) by taking the squeezing parameter zero $(\mathrm{r}=0)$ then Eq. (36) takes the form;

$s=1-\Delta a_{ \pm}^{2}$.

With the help of Eq. (34) we can set quadrature squeezing as;

$s=1-\Delta a_{+}^{2}$.

\section{Conclusions}

In this paper we determine the quantum nature of DSVS, with help of Q function we calculate mean, variance of photon number, quadrature variance and quadrature squeezing. And we find the mean is greater than variance which shows the supperposonity of the system and squeezing occurs in plus quadrature. The other is described in the bellow table.

\begin{tabular}{|l|l|l|l|}
\hline Squeezed parameter(r) & \multicolumn{1}{|c|}{$\Delta a_{+}{ }^{2}$} & Quadrature squeezing(S) & Degree of squeezing \\
\hline 1.5 & 0.049 & 0.95 & $95 \%$ \\
\hline 2 & 0.0183 & 0.981 & $98.1 \%$ \\
\hline 2.5 & 0.0067 & 0.993 & $99.3 \%$ \\
\hline 3 & 0.0024 & 0.997 & $99.7 \%$ \\
\hline
\end{tabular}

Table 1: Shows relation between Squeezed parameter $(r), \Delta a_{+}{ }^{2}$, Quadrature squeezing $(S)$ and Degree of squeezing.

From table 1 we can investigate as squeezing parameter increase quadrature variance decrease but quadrature squeezing(S) increase. And we gate the maximum degree of squeezing to be $99.7 \%$ for $r=3$.

\section{References}

[1] K. Iwasawa, K. Makino, H. Yonezawa, M. Tsang, A. Davidovic, E. Huntington, and A. Furusawa, (2013). Phys. Rev. Lett. 111, 163602

[2] M. A. Taylor, J. Janousek, V. Daria, J. Knittel, B. Hage, H.-A. Bachor, and W. P. Bowen, Nat. (2013). 
Photonics 7, 229 .

[3] Fesseha Kassahun, (2008). Fundamentals of Quantum Optics (Lulu, North Carolina,).

[4] Jinhyoung Leec and M. S. Kim, (2004). On the Disentangling Process for Two-Mode Squeezed State, Journal of the Korean Physical Society, Vol. 44, No. 3, , pp. 691696.

[5] Ts. Gantsog and R.Tanas, (Jaunary 1991). Phase properties of two-mode squeezed vacuum States, physics letters a, vol. 152, No 5,6,

[6] Beyene Abiti ,(2011). Superposed degenerate three level lasers, MSc Thesis(Addis Ababa University,).

[7 ] Ulrik ,( (2016). 30 years of squeezed light generation, Phys. Scr. 91053001 (11pp).

[8] A.S. Daoud, (March 2002). The average number of photons present in a single-mode squeezed state as function of the mean free path,(Department of Mathematics, Faculty of Sciences, University of Zagazig, Zagazig 44519, Egypt,).

[9] Hong-Yi, F., et al., Photon distribution of a squeezed chaotic state. Chinese Physics Letters, 2011. 28(4): p. 040302.

[10] Alemayehu Getahun, Statistical and Squeezing Proprieties of Superposed Single-Mode Squeezed Chaotic State, Advances in Physics Theories and Applications, Vol.78, 2019.

[11] Sitotaw Eshete, Quantum Nature of Light in Displaced Squeezed Chaotic State,vol.69,(2018).

[12] Assamen Ayalew and Fesseha kassahun, Superposed Two mode chaotic, coherent, and Squeezed Vacuum State (Aksum University and Addis AbabaUniversity, July 3,2014). 